\section{Un dialogue précoce indispensable entre cellules MAIT et microbiote}

Hélène Gilgenkrantz
Centre de recherche sur I'Inflammation, Inserm UMR 1149 - Université Paris Diderot, Faculté de médecine Xavier-Bichat, 16 rue Henri Huchard, 75018 Paris, France. helene.gilgenkrantz@inserm.fr
> Les cellules MAIT (mucosal-associated invariant $T$ cells) se situent au carrefour de l'immunité, de l'inflammation, et du microenvironnement microbien. Fortement représentées dans les tissus jouant un rôle de barrière comme le chorion (lamina propria) de la muqueuse intestinale ou la peau, elles sont rapidement activées au contact des bactéries. Elles sont également abondantes dans le sang ou le foie, où elles représentent respectivement de 10 à plus de $20 \%$ des cellules T [1, 2]. Si l'abondance des MAIT est très variable d'un individu à l'autre, leur nombre est en revanche très souvent diminué dans le sang de patients présentant une maladie à $(\rightarrow)$ Voir les Nouvelles composante immu- de I. Magalhaes et nitaire ou inflam- A. Lehuen, $m / s n^{\circ} 8-9$, août-septembre matoire comme le 2015, page 717, et de diabète $[3,4,14]$ 0. Rouxel et $A$. Lehuen, $(\rightarrow)$, la cirrhose $m / s n^{\circ} 6-7$, juin-juillet [5], ou certaines 2018, page 518 maladies auto-immunes [6].

$\varepsilon$ n se fondant sur l'importance de la colonisation intestinale dans le développement du système immunitaire, une équipe américaine s'est interrogée sur le rôle de la flore commensale dans l'abondance et la fonction des lymphocytes MAIT. La faible représentation des cellules MAIT chez la souris et l'absence d'anticorps monoclonal dirigé contre leur récepteur de l'antigène ( $T$ cell receptor, $T C R$ ) rendaient la tâche ardue. L'équipe de Yasmine Belkaid (Bethesda, ÉtatsUnis) vient néanmoins de montrer dans la revue Science que l'exposition intestinale à certaines espèces de bactéries commensales durant une fenêtre de temps limitée aux trois premières semaines de vie postnatale déterminait le développement et l'abondance des cellules MAIT à l'âge adulte, et que les interactions que ces cellules entretiennent avec la flore bactérienne modulaient leur fonction et avaient une influence sur la réparation tissulaire [7].

\section{Éducation du système immunitaire : le} rôle du microbiote

Tous les tissus ayant un rôle de barrière sont colonisés par des bactéries qui permettent la maturation et l'homéostasie du système immunitaire, notamment en relarguant des métabolites microbiens [3]. En retour, le système immunitaire est capable de moduler le microbiote et de maintenir l'intégrité de la fonction barrière du tissu. Ainsi, la colonisation néonatale des poumons permet à la souris adulte d'induire des lymphocytes $T$ régulateurs et de promouvoir une tolérance à certains allergènes [8]. De même, la colonisation intestinale postnatale précoce chez la souris inhibe la prolifération des lymphocytes $T$ «natural killer» (cellules NKT) et prévient le développement d'une colite induite [9-10]. Néanmoins, la nature des antigènes et des signaux impliqués dans ce dialogue restait encore mystérieuse, et le rôle même des cellules immunitaires dans I'homéostasie tissulaire était mal compris.

\section{Nature et fonction des cellules MAIT}

Les cellules MAIT ont été décrites il y a presque 20 ans par l'équipe française d'0. Lantz [11]. Elles appartiennent à la famille des lymphocytes $T$ non conventionnels et ont, à l'instar des cellules
iNKT (invariant natural killer T cells), la particularité d'exprimer un récepteur T semi-invariant puisque la chaîne invariante $\alpha$ (V $\alpha 7.2-J \alpha 33$ chez l'homme et V $\alpha 19$-J $\alpha 33$ chez la souris) s'associe à un nombre restreint de chaînes $\beta$. La proportion des lymphocytes MAIT sanguins augmente au cours du développement post-natal, atteint un pic à l'âge adulte, puis a tendance à diminuer avec l'âge.

La maturation des MAIT est dépendante de la molécule non polymorphe MRl (major histocompatibility complex class I-related protein) de classe 1 b du système majeur d'histocompatibilité, qui est exprimée par différents types de cellules. Si l'expression de MRl est nécessaire au développement des MAIT - les souris déficientes en MRl en sont en effet dépourvues -, en revanche elle n'est pas indispensable à leurs fonctions effectrices. Il aura néanmoins fallu attendre l'année 2012 pour comprendre que les cellules présentatrices d'antigènes (CPA) utilisaient MRI pour présenter aux cellules MAIT des antigènes microbiens particuliers, issus de la voie de synthèse de la vitamine B2 (riboflavine) [12], qui sont hautement conservés dans différentes espèces de bactéries (entérobactéries, staphylocoques, mycobactéries, etc.), champignons et levures.

Les cellules MAIT ont une fonction effectrice mémoire, et sont activées de deux façons différentes: soit de façon dépendante de la présentation d'antigènes microbiens par MRl, soit de façon MRlindépendante, via certaines cytokines inflammatoires comme les interleukines (IL) 12 et 18 (Figure 1). Elles répondent alors très rapidement à ces stimulations 


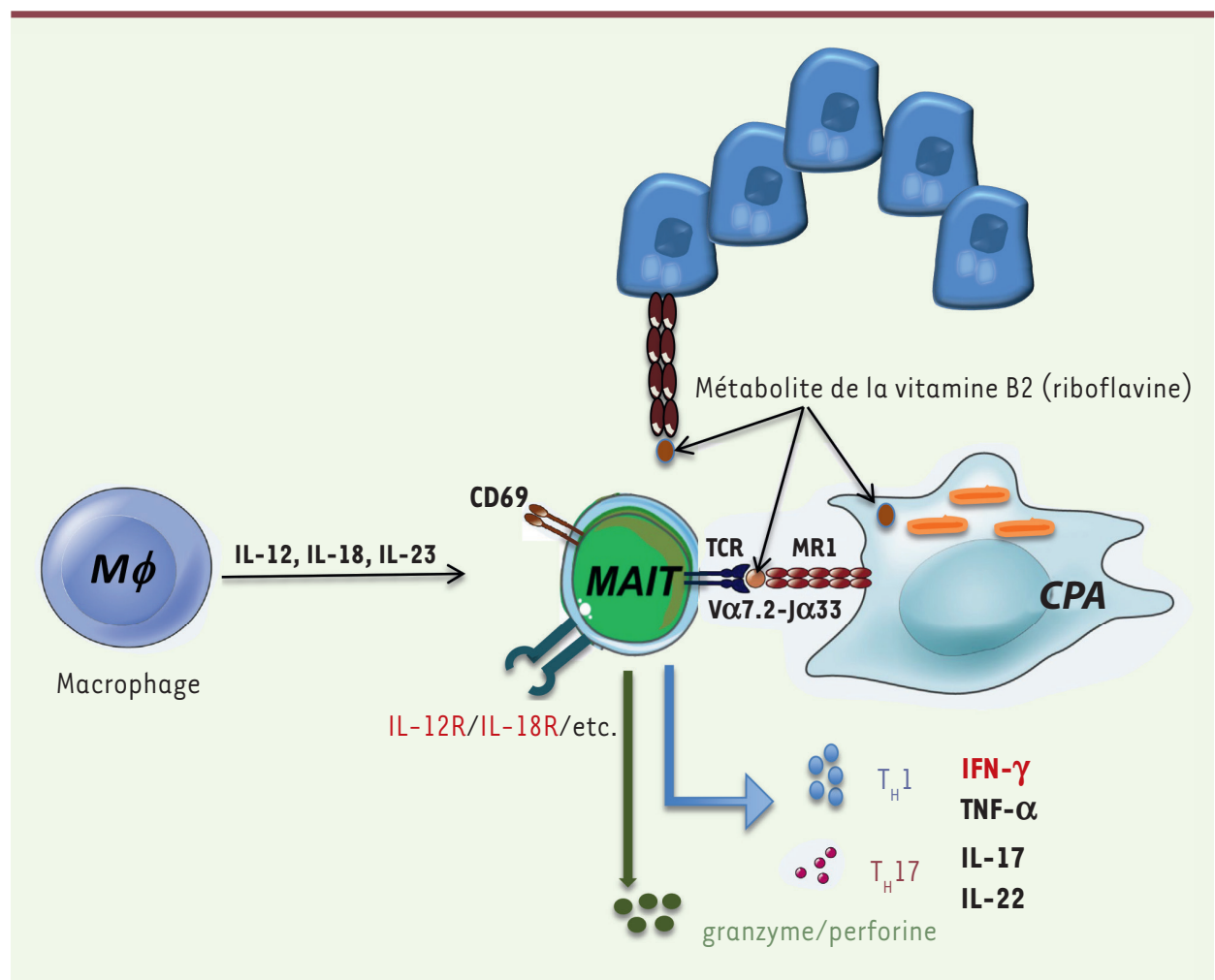

Figure 1. Mécanismes d'activation des MAIT. Les lymphocytes MAIT sont activés par deux voies : (1) par certaines cytokines sécrétées par les monocytes/macrophages ou par des cellules épithéliales (IL-12, IL-18, IL-23) ou (2) via la molécule MRI du complexe majeur d'histocompatibilité qui active le TCR semi-invariant des MAIT. Au cours d'une infection par des pathogènes, les cellules «professionnelles » de la présentation d'antigènes (CPA) ou les cellules épithéliales peuvent présenter les métabolites bactériens comme la vitamine B2 (ou riboflavine) via la molécule MRl. En retour, les cellules MAIT produiront des cytokines inflammatoires de type $T_{H} 1$ ou $T_{H} 17$ et auront également des fonctions cytotoxiques en sécrétant granzyme et perforine.

en sécrétant des cytokines pro-inflammatoires de profil $T_{H} 1 / T_{H} 17$, notamment l'interféron- $\gamma($ IFN $-\gamma)$, le tumor necrosis factor- $\alpha$ (TNF- $\alpha)$, I'IL-17 ou I'IL-22, et ont aussi des fonctions cytotoxiques puisqu'elles libèrent du granzyme $B$ et de la perforine lorsqu'elles sont activées. Leur capacité sécrétoire varie en fonction des tissus. La contribution respective des antigènes et des cytokines pour activer les MAIT est néanmoins encore mal comprise.

Homéostasie tissulaire des MAIT : une fenêtre étroite dans le développement post-natal

Comme les autres lymphocytes T, les MAIT se développent dans le thymus, où elles sont immatures, et acquièrent leurs fonctions effectrices pendant leur développement [13]. Elles s'accumulent dans les tissus barrières entre 2 et 3 semaines de vie chez la souris (Figure 2). Or, si leur abondance tissulaire est très variable au sein d'une même lignée de souris élevées en conditions dépourvues de pathogènes spécifiques, cette disparité disparaît lorsque les souris sont élevées dans une même cage, suggérant le rôle potentiel du microbiote dans cette variabilité (rappelons que les souris sont coprophages, ce qui entraîne une homogénéisation de leur microbiote au sein d'un même élevage). Pour le montrer, les auteurs ont étudié la corrélation entre l'apparition de bactéries commensales dans l'intestin et la dynamique d'apparition et de maturation des MAIT dans la peau, où elles représentent jusqu'à $40 \%$ des lymphocytes T $\alpha \beta$ chez la souris adulte et $2 \%$ des lymphocytes $\mathrm{CD}^{+}$chez l'homme. En colonisant des souris dépourvues de microbiote (souris germ-free, GF, ou axéniques) à différents temps après la naissance par les cinq espèces de bactéries qui sont normalement présentes à cette période de la vie, les auteurs ont démontré que le développement et l'accumulation des MAIT dans les tissus nécessitaient une exposition aux germes durant les trois premières semaines post-natales. Passé ce délai, si les lymphocytes $T$ helper $T_{H} 17$ sont toujours induits, il existe en revanche un défaut de représentation et d'activation des cellules MAIT. Parmi ces cinq espèces bactériennes, Proteus mirabilis suffit à permettre le développement des MAIT. Ainsi, il est possible de reproduire localement l'exposition aux antigènes microbiens pendant une période, toutefois restreinte, du développement post-natal, faute de quoi le développement et la maturation des cellules MAIT seront définitivement compromis [7].

\section{Activation et rôle des MAIT cutanées dans la réparation tissulaire}

Les auteurs confirment que les cellules MAIT sont activées localement, soit par des cytokines, notamment I'IL-l et I'IL18 , soit par une reconnaissance d'antigènes, métabolites de la riboflavine, par l'intermédiaire de MRl. Les résultats obtenus suggèrent que, dans le cadre d'une réponse homéostatique au microbiote, l'activation des MAIT est strictement dépendante du TCR (Figure 2). Dans la peau, les MAIT sont présentes à l'interface entre le derme et l'épiderme, avec une majorité dans le derme et à proximité de la couche basale de l'épiderme. L'analyse du profil trans- 


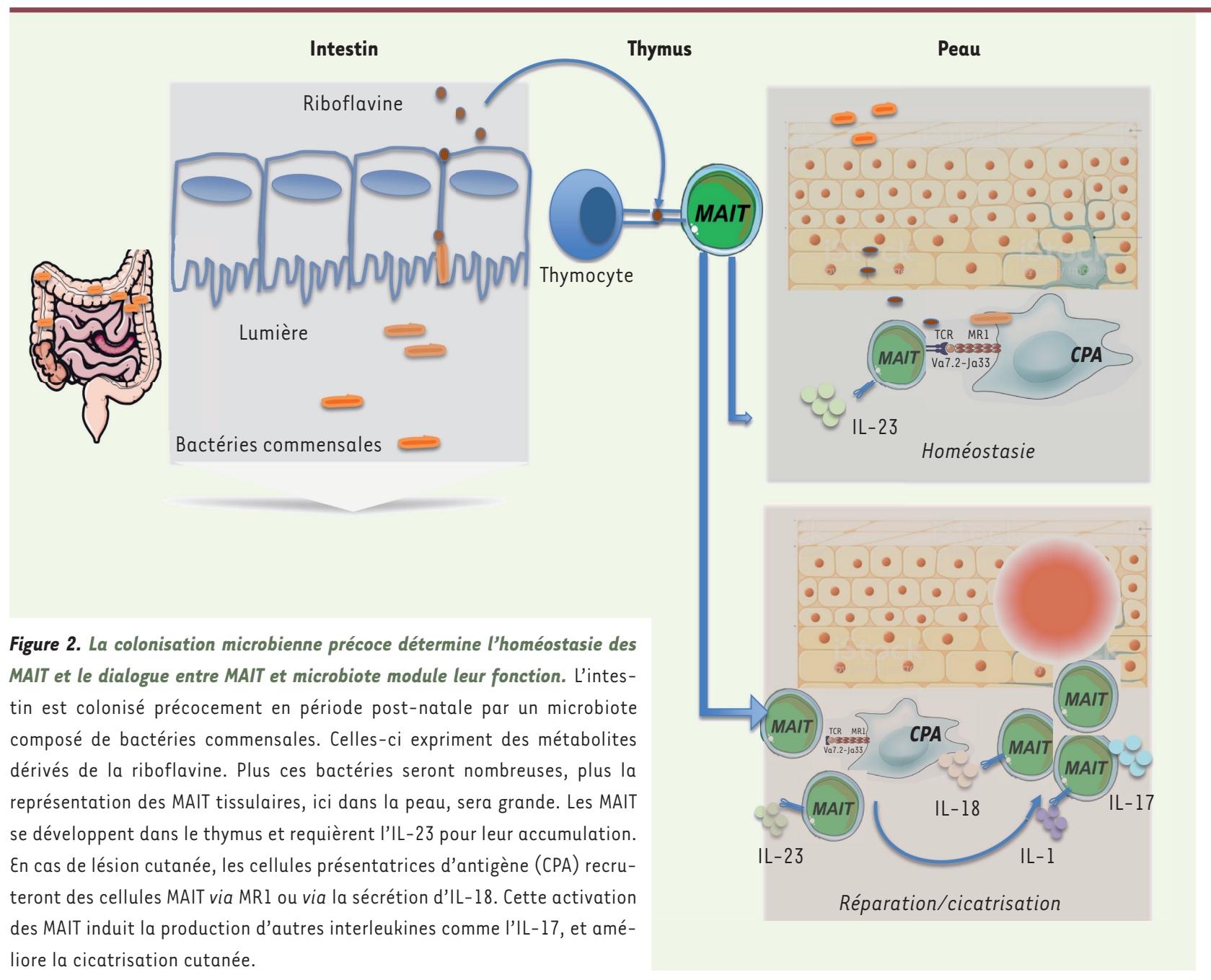

criptionnel des MAIT cutanées révèle l'expression de nombreux gènes de réparation tissulaire. Les MAIT cutanées sont activées localement par les antigènes microbiens de la flore commensale. Pour déterminer si, à l'instar d'autres lymphocytes ILC (innate-like cells), les MAIT ont un rôle dans la réparation tissulaire, un modèle de re-épithélialisation après lésion cutanée a été développé chez des souris rendues déficientes en MAIT par invalidation du gène codant MRI (souris $\mathrm{MrI}^{-/-}$). Ces souris cicatrisent moins rapidement que des souris témoins. Ce rôle est également souligné par le fait que l'administration locale d'un antigène microbien dérivé de la riboflavine induit un afflux de cellules MAIT cutanées et améliore la réparation et la cicatrisation [7] (Figure 2). La plupart des bactéries commensales de la peau exprimant des métabolites dérivés de la riboflavine, ces antigènes pourraient contribuer non seulement à l'homéostasie cutanée, mais également à la réparation tissulaire en cas de lésion.

Ce travail permet de mieux comprendre le dialogue qui se met en place très précocement au cours de la vie des mammifères entre le système immunitaire et le microbiote, via la reconnaissance d'antigènes microbiens spécifiques qui contrôleraient l'homéostasie des MAIT. Il montre également que la fenêtre temporelle post-natale permettant au microbiote, en particulier aux bactéries qui synthétisent la riboflavine, de déterminer le nombre et l'activité des MAIT est restreinte, au moins chez la souris. Cette observation pourrait expli- quer l'abondance très variable des MAIT notée chez l'homme. Néanmoins, dans l'espèce humaine, les lymphocytes $T$ se développent in utero, et l'empreinte pourrait se produire avant la naissance. La participation des MAIT à la réparation tissulaire ouvre des perspectives thérapeutiques intéressantes puisque l'on peut désormais imaginer que l'administration topique ${ }^{1}$ de certains antigènes pourrait accélérer la cicatrisation. $\diamond$ An early crosstalk between MAIT cells and microbiota is required for tissue homeostasis and repair

\footnotetext{
${ }^{1}$ Forme d'administration d'un médicament sur un point externe du corps, sur une surface du corps telle que la peau ou les muqueuses.
}

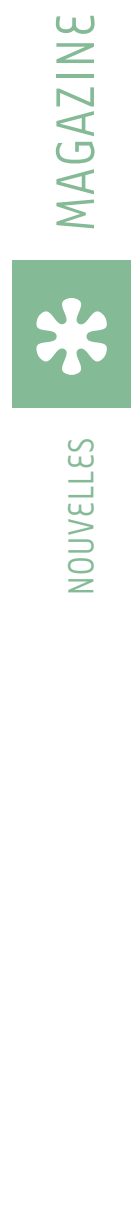




\section{LIENS D'INTÉRÊT}

L'auteure déclare n'avoir aucun lien d'intérêt concernant les données publiées dans cet article.

\section{RÉFÉRENCES}

1. Chen P, Deng W, Li D, et al. Circulating mucosalassociated invariant $\mathrm{T}$ cells in a large cohort of healthy chinese indiviuals from newborn to elderly. Front Immunol 2019; $10: 260$

2. Ben Youssef G, Tourret M, Salou M, et al. Ontogeny of human mucosal-associated invariant T cells and related T cell subsets. J Exp Med 2018 ; 215 : 459-79.

3. Belkaid $y$, Hand TW. Role of the microbiota in immunity and inflammation. Cell 2014 ; 157 : 121-41.

4. Magalhaes I, Lehuen A. Les lymphocytes MAIT dans le diabète de type 2 et l'obésité. Med Sci (Paris) 2015 ; $31: 717-9$.
5. Hegde P, Weiss $\varepsilon$, Paradis V, et al. Mucosal-invariant $T$ cells are a profibrogenic immune cell population in the liver. Nat Commun $2018 ; 9: 2146$.

6. Toubal A, Nel I, Lotersztajn S, Lehuen A. Mucosalassociated invariant T cells and disease. Nat Rev Immunol 2019 ; 19 : 643-57.

7. Constantinedes MG, Link VM, Tamoutounour $S$, et al. MAIT cells are imprinted by the microbiota in early life and promote tissue repair. Science $2019 ; 366: 1-13$.

8. Gollwitzer ES, Saglani S, Trompette A, et al. Lung microbiota promotes tolerance to allergens in neonates via PDL-1. Nat Med $2014 ; 20: 642-7$.

9. Olszak T, An D, Zeissig S, et al. Microbial exposure during early life has persistent effects on natural killer T cell function. Science 2012 ; $336: 489-93$.

10. An D, Oh SF, Olszak T et al. Sphingolipids from a symbiotic microbe regulate homeostasis of host intestinal natural killer T cells. Cell 2014 ; 156 : 123-33.
11. Treiner $\varepsilon$, Duban L, Bahram S, et al. Selection of evolutionarily conserved mucosal-associated invariant T cells by MR1. Nature $2003 ; 422$ : 164-9.

12. Kjer-Nielsen $L$, Patel 0 , Corbett AJ, et al. MRA présents microbial vitamin B métabolites to MAIT cells. Nature $2012 ; 491: 717-23$

13. Koay HF, Gherardin NA, Enders A, et al. A three stage intrathymic development pathway for the mucosalassociated invariant T cell lineage. Nat Immunol $2016 ; 17: 1300-11$

14. Rouxel 0 , Lehuen A. Les cellules MAIT : un lien entre l'intestin et le diabète de type 1. Med Sci (Paris) 2018 ; 34 : 518-22. 